\title{
Obesity management in polycystic ovary syndrome: disparity in knowledge between obstetrician-gynecologists and reproductive endocrinologists in China
}

\author{
Ruilin Ma ${ }^{1}$, Ying Zou ${ }^{2}$, Wei Wang ${ }^{3}$, Qingmei Zheng ${ }^{4}$, Ying Feng ${ }^{5}$, Han Dong ${ }^{6}, Z^{2}$ hangyun Tan ${ }^{7}$, Xiaoqin Zeng ${ }^{8}$,
} Yinqing Zhao ${ }^{7}$, Yan Deng ${ }^{1}$, Yanfang Wang ${ }^{1}$ and Aijun Sun ${ }^{1,9^{*}}$ (B)

\begin{abstract}
Background: Obesity is associated with the development of polycystic ovary syndrome (PCOS) and contributes substantially to metabolic abnormalities in women with PCOS. The study aimed to describe and compare the practices of physicians in the diagnosis, evaluation, and treatment of obesity in patients with PCOS.

Methods: Reproductive endocrinologists (Repro-Endo) and obstetrician-gynecologists (non-reproductive medicine specialty, OB-Gyn) in China participated in a survey, and their responses were analyzed using $x^{2}$ tests, Fisher exact tests, and multivariable logistic regression analysis.

Results: The study analyzed 1318 survey responses (85.8\% OB-Gyn; 97.3\% women). Body mass index was the most common diagnostic criterion for obesity; only $1.3 \%$ of participants measured waist circumference to identify abdominal obesity. More Repro-Endo participants (25\% of all participants) enquired about the psychological problems of patients with obesity than OB-Gyn participants, and $42.5 \%$ of participants reported ordering both a lipid profile and oral glucose tolerance test (OGTT) for patients with obesity and PCOS. Multivariable analysis, that included physician's specialty, age, hospital grade, and number of patients with PCOS seen annually, revealed that OB-Gyn participants were less likely to order OGTT $(\mathrm{OR}, 0.3 ; 95 \% \mathrm{Cl}, 0.2-0.4)$ and lipid profile $(\mathrm{OR}, 0.2 ; 95 \% \mathrm{Cl}, 0.1-$ 0.3) than Repro-Endo participants. The most common treatments for patients with PCOS were lifestyle modification (>95\%) and metformin (> 80\%). More Repro-Endo participants prescribed metformin at a dose of $1.5 \mathrm{~g} /$ day compared with OB-Gyn (47.6\% vs. 26.3\%), and more OB-Gyn participants reported being unclear about the appropriate dosage of metformin for patients with obesity and PCOS (8.9\% vs. 1.6\%).

Conclusion: Our survey identified knowledge gaps in metabolic screening for patients with obesity and PCOS and a disparity in the evaluation and treatment of obesity in PCOS among different specialties. Similarly, it highlights the need to improve obesity management education for physicians caring for women with PCOS.
\end{abstract}

Keywords: Obesity, Polycystic ovary syndrome, Oral glucose tolerance test, Lipid profile, Metformin

\footnotetext{
*Correspondence: saj_pumch@sina.com

'Department of Obstetrics and Gynecology, Peking Union Medical College Hospital, Chinese Academy of Medical Sciences and Peking Union Medical College, Beijing 100730, China

${ }^{9}$ Department of Obstetrics and Gynecology, Peking Union Medical College Hospital, No. 1 Shuaifuyuan, Dongcheng District, Beijing 100010, China

Full list of author information is available at the end of the article
}

(c) The Author(s). 2021 Open Access This article is licensed under a Creative Commons Attribution 4.0 International License, which permits use, sharing, adaptation, distribution and reproduction in any medium or format, as long as you give appropriate credit to the original author(s) and the source, provide a link to the Creative Commons licence, and indicate if changes were made. The images or other third party material in this article are included in the article's Creative Commons licence, unless indicated otherwise in a credit line to the material. If material is not included in the article's Creative Commons licence and your intended use is not permitted by statutory regulation or exceeds the permitted use, you will need to obtain permission directly from the copyright holder. To view a copy of this licence, visit http://creativecommons.org/licenses/by/4.0/ The Creative Commons Public Domain Dedication waiver (http://creativecommons.org/publicdomain/zero/1.0/) applies to the data made available in this article, unless otherwise stated in a credit line to the data. 


\section{Background}

According to the results of the China Hypertension Survey $(\mathrm{CHS})$, a nationally representative cross-sectional study among residents aged $\geq 18$ years that was conducted from October 2012 to December 2015, more than two-fifths of Chinese adults were obese or overweight [1]. A high body mass index (BMI) and an unhealthy diet are the main risk factors for chronic diseases among Chinese residents [2]. Both being overweight and obese are associated with multiple comorbidities, including metabolic syndrome, type 2 diabetes mellitus (T2DM), cancer, cardiovascular diseases, and obstructive sleep apnea, and these may lead to mortality.

Polycystic ovary syndrome (PCOS) is a well-known endocrine/metabolic disorder that affects females of different age groups, including the age of puberty, childbearing, and perimenopause. Although the pathogenesis of PCOS remains unclear, the relationship between heredity and environmental factors may play a significant role in its development [3]. As one of the environmental factors, obesity, caused by unbalanced food intake and expenditure, and exposure to environmental chemicals disrupting endocrine functions during critical growth stages, contributes substantially to endocrine and metabolic disorders in patients with PCOS [4]. Approximately $37 \%$ of women with PCOS are obese or overweight in China [5]; furthermore, women with PCOS have more visceral fat than age-matched and BMI-matched controls [6]. Obesity, especially abdominal obesity, has been reported to aggravate insulin resistance (IR), hyperandrogenism, and dyslipidemia, and increase the risk for T2DM, impaired glucose tolerance (IGT), cardiovascular diseases, non-alcoholic fatty liver disease, and psychological problems in women with PCOS [6-8]. Therefore, prompt recognition and management of obesity and related metabolic complications in women with PCOS are crucial. The Chinese Medical Association, American College of Obstetricians and Gynecologists (ACOG), and international evidence-based guidelines have suggested that all patients with PCOS should be assessed for diabetes and cardiovascular risk factors, including BMI, waist circumference, oral glucose tolerance test (OGTT), and lipid profile, and lifestyle modification should be recommended in all those with PCOS to enhance the quality of life and modify risks for cardiovascular disease and diabetes [9-11].

Several international online surveys have focused on practices regarding the diagnosis and management of PCOS among physicians [12-14]. However, data on the management of obesity and metabolic screening in patients with PCOS are scarce. In addition, previous physician surveys showed differences in practice patterns between gynecologists and reproductive endocrinologists in the United States [14], as well as between gynecologists and medical endocrinologists in Australia [15]. Therefore, we surveyed reproductive endocrinologists (Repro-Endo) and obstetrician-gynecologists (nonreproductive medicine specialty, OB-Gyn) on their usual practice regarding the diagnosis, evaluation, and treatment of obesity in patients with PCOS. Our study aimed to promote evidence-based care, patient satisfaction, and minimize long-term morbidities in women with PCOS.

\section{Methods}

The study was approved by the Ethics Committee of Peking Union Medical College Hospital, Chinese Academy of Medical Sciences (No. S-K1373).

\section{Study design, setting, and participants}

The study was a nationwide, online survey conducted from September 1, 2020 to September 30, 2020. Physicians registered in online chat groups of the China Maternal and Child Health Association received an invitation to complete this questionnaire. Information about the questionnaire and the URL of the online survey was contained in the message. Participation was voluntary and anonymous. Completion of the survey was taken as consent to participate.

\section{Questionnaire content}

The questionnaire included the following data: provider's demographics, clinical specialty, hospital grade, practice characteristics, diagnostic criteria used for obesity, provider's knowledge of evaluation and treatment of women with obesity and PCOS, and practices regarding weight loss in women with obesity and PCOS. The questionnaire was piloted with 30 obstetricians and gynecologists, and their feedback was incorporated; there was no ambiguity or doubt regarding the contents of the questionnaire. The full questionnaire details are provided in Supplementary material.

\section{Statistical analysis}

Statistical analysis was performed using SPSS version 26.0 software (IBM, Armonk, NY, USA). Categorical data are presented as frequencies and percentages, and groups were compared using Pearson's $\chi^{2}$ tests or Fisher's exact tests when appropriate. Variables included in the multivariable logistic regression analysis were as follows: physician's specialty, age, hospital grade, number of patients with PCOS seen annually, and proportion of patients with obesity and PCOS. A $p$-value of $<0.05$ was considered statistically significant.

\section{Results}

In total, 1415 questionnaires were completed and submitted. Questionnaires filled by non-obstetricians and 
gynecologists $(n=97)$ were excluded, and 1318 questionnaires were included in the final analysis.

\section{General characteristics of the participants}

Table 1 summarizes the general characteristics of the 1318 participants, including 14.2\% Repro-Endo and 85.8\% OB-Gyn participants. Most participants were female and above 35 years of age. About $60 \%$ of the participants were from tertiary hospitals, and 34.6\% were from secondary hospitals. More OB-Gyn participants saw fewer than 50 patients with PCOS per year, and more Repro-Endo participants saw 50-200 patients or more than 200 patients with PCOS per year. Most participants in the OB-Gyn group (71.6\%) believed that the prevalence of overweight/obesity was less than $50 \%$ in patients with PCOS, while $80.7 \%$ of participants in the ReproEndo group believed that the prevalence of overweight/ obesity was $50-80 \%$. Menstrual disorder (80.6\%) was the most common reason for patient's visit, followed by infertility (17.1\%). Participants in the OB-Gyn group saw more patients with menstrual disorders, and the Repro-
Endo participants saw more patients with infertility. A few patients with PCOS (1.6\%) consulted a doctor primarily for obesity or IR, and fewer patients with PCOS saw a doctor primarily for hirsutism or acne (Table 1).

\section{Assessment of women with obesity and PCOS}

BMI was the most common diagnostic criterion used for obesity. Most participants (64.7\% of Repro-Endo, $58.7 \%$ of OB-Gyn participants, $P=0.122$ ) chose the Chinese criteria (BMI $\geq 24 \mathrm{~kg} / \mathrm{m}^{2}$ for overweight and $\geq 28 \mathrm{~kg} / \mathrm{m}^{2}$ for obesity) when classifying patients' weight. Overall, only $1.3 \%$ of the participants preferred measuring waist circumference to determine abdominal obesity, including 2.7 and $1.1 \%$ of those in the Repro-Endo and OB-Gyn groups, respectively $(P=0.065)$ (Table 2$)$.

Only $25.5 \%$ of the participants inquired about symptoms of anxiety, depression, and other psychological problems in patients with obesity and PCOS. This included 56.1 and $20.4 \%$ of participants in the Repro-Endo and OB-Gyn groups, respectively $(P<0.001$; Table 2$)$.

Table 1 General characteristics of 1318 respondents

\begin{tabular}{|c|c|c|c|c|}
\hline & Overall $(n=1318)$ & Repro-Endo $(n=187)$ & OB-Gyn $(n=1131)$ & $P$-value \\
\hline \multicolumn{5}{|l|}{ Sex } \\
\hline female & $1282(97.3)$ & $177(94.7)$ & $1105(97.7)$ & .018 \\
\hline male & $36(2.7)$ & $10(5.3)$ & $26(2.3)$ & .018 \\
\hline \multicolumn{5}{|l|}{ Age } \\
\hline $18-35$ & $226(17.1)$ & $22(11.7)$ & $204(18.0)$ & .035 \\
\hline $36-45$ & $514(39.0)$ & $76(40.7)$ & $438(38.7)$ & NS \\
\hline$\geq 46$ & $578(43.9)$ & $89(47.6)$ & $489(43.3)$ & NS \\
\hline \multicolumn{5}{|l|}{ Hospital grade } \\
\hline 3 & $793(60.2)$ & $123(65.8)$ & $670(59.2)$ & NS \\
\hline 2 & $456(34.6)$ & $61(32.6)$ & $395(34.9)$ & NS \\
\hline 1 & $69(5.2)$ & $3(1.6)$ & $66(5.9)$ & .016 \\
\hline \multicolumn{5}{|c|}{ No. of patients with PCOS treated annually } \\
\hline$<50$ & $849(64.4)$ & $54(28.8)$ & $795(70.3)$ & $<.001$ \\
\hline $51-200$ & $358(27.2)$ & $86(46.0)$ & $272(24.0)$ & $<.001$ \\
\hline$>200$ & $111(8.4)$ & $47(25.2)$ & $64(5.7)$ & $<.001$ \\
\hline \multicolumn{5}{|c|}{ Main reason for clinic attendance in patients with PCOS } \\
\hline Menstrual disorders & $1062(80.6)$ & $130(69.5)$ & $932(82.4)$ & $<.001$ \\
\hline Infertility & $226(17.1)$ & $54(29.0)$ & $172(15.2)$ & $<.001$ \\
\hline Hirsutism/acne & $9(0.7)$ & $1(0.5)$ & $8(0.7)$ & NS \\
\hline Obesity/IR & $21(1.6)$ & $2(1.0)$ & $19(1.7)$ & NS \\
\hline \multicolumn{5}{|c|}{ Estimated national prevalence of overweight/obesity in patients with PCOS } \\
\hline $0-30 \%$ & $384(29.1)$ & $28(15.0)$ & $356(31.5)$ & $<.001$ \\
\hline $31-50 \%$ & $541(41.1)$ & $87(46.5)$ & $454(40.1)$ & NS \\
\hline $51-80 \%$ & $331(25.1)$ & $64(34.2)$ & $267(23.6)$ & .002 \\
\hline$>80 \%$ & $62(4.7)$ & $8(4.3)$ & $54(4.8)$ & NS \\
\hline
\end{tabular}

Abbreviations: Repro-Endo Reproductive endocrinologist, OB-Gyn Obstetrician-gynecologists, PCOS Polycystic ovary syndrome 
Table 2 Practices of physicians in the management of patients with obesity and PCOS

\begin{tabular}{|c|c|c|c|c|}
\hline & Overall $(n=1318)$ & Repro-Endo $(n=187)$ & OB-Gyn $(n=1131)$ & $P$-value \\
\hline \multicolumn{5}{|c|}{ Commonly used diagnostic criterion for obesity } \\
\hline BMI (WHO criteria) & $353(26.7)$ & $29(15.5)$ & $324(28.6)$ & $<.001$ \\
\hline BMI (Chinese criteria) & $785(59.6)$ & $121(64.7)$ & $664(58.7)$ & NS \\
\hline BMI (Asian criteria) & $164(12.4)$ & $32(17.1)$ & $132(11.7)$ & .037 \\
\hline Measure waistline & $16(1.3)$ & $5(2.7)$ & $12(1.1)$ & NS \\
\hline \multicolumn{5}{|c|}{ Inquiry about family history of diabetes } \\
\hline Yes & $1261(95.7)$ & $180(96.3)$ & $1081(95.6)$ & NS \\
\hline \multicolumn{5}{|c|}{ Inquiry about family history of cardiovascular disease } \\
\hline Yes & $882(66.9)$ & $131(70.1)$ & $751(66.4)$ & NS \\
\hline \multicolumn{5}{|c|}{ Inquiry about family history of PCOS } \\
\hline Yes & $1179(89.5)$ & $168(89.8)$ & $1011(89.4)$ & NS \\
\hline \multicolumn{5}{|c|}{ Inquiry about symptoms of anxiety, depression, and other psychological problems } \\
\hline Yes & $336(25.5)$ & $105(56.1)$ & $231(20.4)$ & $<.001$ \\
\hline
\end{tabular}

Abbreviations: Repro-Endo Reproductive endocrinologist, OB-Gyn Obstetrician-gynecologists, PCOS polycystic ovary syndrome, BMI Body mass index, WHO World Health Organization, NS No significant

The most frequently ordered metabolic screening tests for patients with obesity and PCOS among all the participants were fasting glucose and fasting insulin tests (78.5\%), followed by OGTT (59.8\%) and lipid profile (46.2\%) (Fig. 1). About 2 in 5 participants (42.5\%) reported ordering both lipid profile and OGTT for patients with obesity and PCOS.

In univariate analysis, physicians in secondary and tertiary hospitals, those who saw more than 50 patients with PCOS annually, and those who estimated that the prevalence of overweight/obesity was $51-80 \%$ were more likely to order OGTT for patients with obesity and PCOS. Physicians who saw 51-200 patients with PCOS annually and estimated the prevalence of overweight/ obesity as $51-80 \%$ were more likely to order a lipid profile test for patients with obesity and PCOS (Table 3 ). Multivariate regression analysis revealed that participants in the OB-Gyn group were less likely to order an OGTT (odds ratio (OR) $0.3,95 \%$ confidence interval (CI) $0.2-0.4$ ) and a lipid profile test (OR 0.2; 95\% CI 0.1-0.3) (Table 3) than those in the Repro-Endo group.

\section{Management of women with obesity and PCOS}

When asked about the commonly prescribed treatments for patients with obesity and PCOS with or without fertility requirements, the participants' first choice was

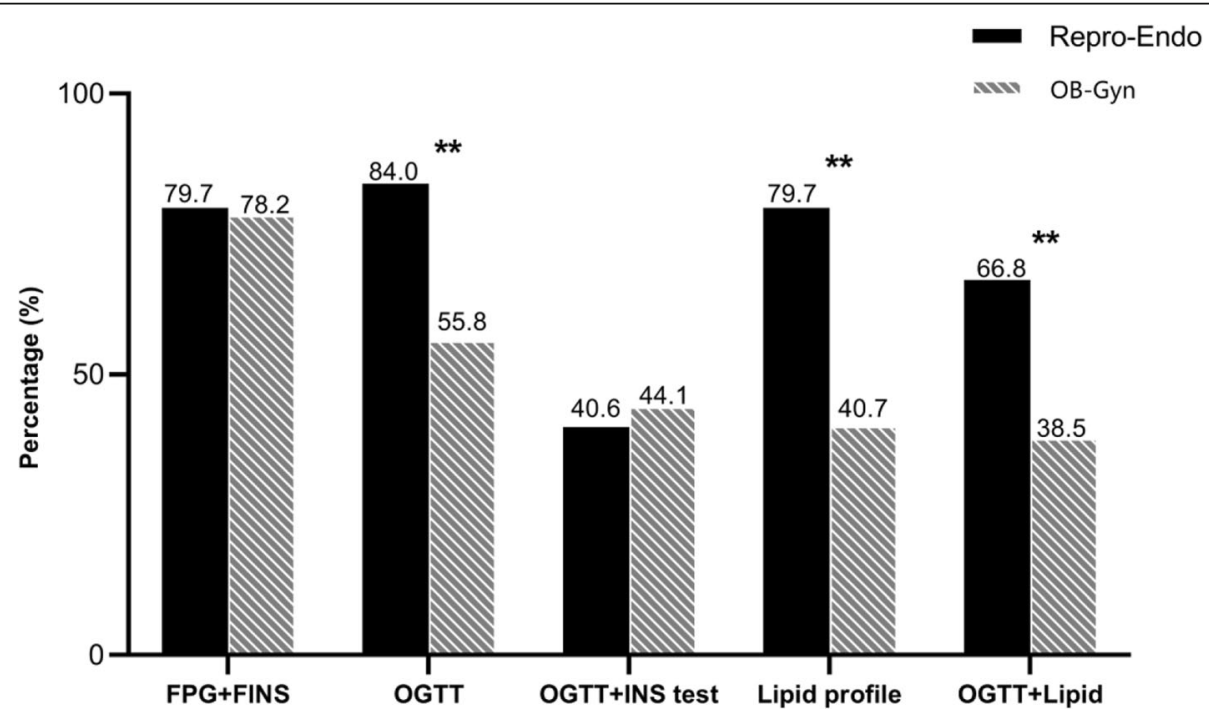

Fig. 1 Metabolic screening tests used for patients with obesity and PCOS by Repro-Endo and OB-Gyn. Repro-Endo, reproductive endocrinologist; OB-Gyn, obstetrician-gynecologists; FPG, fasting plasma glucose; FINS, fasting insulin; OGTT, oral glucose tolerance test. ${ }^{*} p<0.05,{ }^{*} p<0.001$ 
Table 3 Independent associations between physician characteristics and likelihood of physicians ordering OGTT and lipid profiles for patients with PCOS using multivariable logistic regression analysis

\begin{tabular}{|c|c|c|c|c|c|c|c|c|}
\hline Variables & $\begin{array}{l}\text { Physicians } \\
\text { ordering OGTT }\end{array}$ & $\begin{array}{l}\text { Physicians not } \\
\text { ordering OGTT }\end{array}$ & Crude & Adjusted & $\begin{array}{l}\text { Physicians ordering } \\
\text { lipid profiles }\end{array}$ & $\begin{array}{l}\text { Physicians not ordering } \\
\text { lipid profiles }\end{array}$ & $\begin{array}{l}\text { Crude } \\
\text { OR }\end{array}$ & $\begin{array}{l}\text { Adjusted } \\
\text { OR }\end{array}$ \\
\hline \multicolumn{9}{|l|}{ Age } \\
\hline $18-35$ & $5(0.6)$ & $3(0.6)$ & 1 & 1 & $4(0.7)$ & $4(0.6)$ & 1 & 1 \\
\hline $36-45$ & $121(15.4)$ & $97(18.3)$ & $\begin{array}{l}0.8(0.2, \\
3.2)\end{array}$ & $\begin{array}{l}0.8(0.2 \\
3.3)\end{array}$ & $101(16.5)$ & $117(16.5)$ & $\begin{array}{l}0.9(0.2, \\
3.5)\end{array}$ & $\begin{array}{l}0.9(0.2, \\
3.8)\end{array}$ \\
\hline$\geq 46$ & $662(84.0)$ & $430(81.1)$ & $\begin{array}{l}0.9(0.2 \\
3.9)\end{array}$ & $\begin{array}{l}0.9(0.2, \\
3.8)\end{array}$ & $504(82.8)$ & $588(82.9)$ & $\begin{array}{l}0.9(0.2, \\
3.5)\end{array}$ & $\begin{array}{l}0.8(0.2, \\
3.4)\end{array}$ \\
\hline \multicolumn{9}{|c|}{ Hospital grade } \\
\hline 1 & $31(3.9)$ & $38(7.2)$ & 1 & 1 & $29(4.8)$ & $40(5.6)$ & 1 & 1 \\
\hline 2 & $263(33.4)$ & $193(36.4)$ & $\begin{array}{l}1.7(1.0 \\
2.8)^{\mathbf{a}}\end{array}$ & $\begin{array}{l}1.4(0.9 \\
2.4)\end{array}$ & $203(33.3)$ & $253(35.7)$ & $\begin{array}{l}1.1(0.7 \\
1.8)\end{array}$ & $\begin{array}{l}0.9(0.5 \\
1.6)\end{array}$ \\
\hline 3 & $494(62.7)$ & $299(56.4)$ & $\begin{array}{l}2.0(1.2 \\
3.3)^{\mathbf{a}}\end{array}$ & $\begin{array}{l}1.7(1.0 \\
2.8)^{\mathbf{a}}\end{array}$ & $377(61.9)$ & $416(58.7)$ & $\begin{array}{l}1.3(0.8, \\
2.1)\end{array}$ & $\begin{array}{l}1.0(0.6 \\
1.7)\end{array}$ \\
\hline \multicolumn{9}{|l|}{ Specialty } \\
\hline $\begin{array}{l}\text { Repro- } \\
\text { Endo }\end{array}$ & $157(19.9)$ & $30(5.7)$ & 1 & 1 & $149(24.5)$ & $38(5.4)$ & 1 & 1 \\
\hline $\begin{array}{l}\text { OB- } \\
\text { Gyn }\end{array}$ & $631(80.1)$ & $500(94.3)$ & $\begin{array}{l}0.2(0.2 \\
0.4)^{a}\end{array}$ & $\begin{array}{l}0.3(0.2 \\
0.4)^{\mathbf{a}}\end{array}$ & $460(75.5)$ & $671(94.6)$ & $\begin{array}{l}0.2(0.1 \\
0.3)^{\mathbf{a}}\end{array}$ & $\begin{array}{l}0.2(0.1 \\
0.3)^{\mathbf{a}}\end{array}$ \\
\hline \multicolumn{9}{|c|}{ Annual patients with PCOS } \\
\hline$<50$ & $469(59.5)$ & $380(71.7)$ & 1 & 1 & $364(59.8)$ & $485(68.4)$ & 1 & 1 \\
\hline $51-200$ & $242(30.7)$ & $116(21.9)$ & $\begin{array}{l}1.7(1.3 \\
2.2)^{\mathbf{a}}\end{array}$ & $\begin{array}{l}1.3(1.0 \\
1.8)\end{array}$ & $188(30.9)$ & $170(24.0)$ & $\begin{array}{l}1.5(1.2, \\
1.9)^{\mathbf{a}}\end{array}$ & $\begin{array}{l}1.1(0.9 \\
1.5)\end{array}$ \\
\hline$>200$ & $77(9.8)$ & $34(6.4)$ & $\begin{array}{l}1.8(1.2 \\
2.8)^{\mathbf{a}}\end{array}$ & $\begin{array}{l}1.2(0.7 \\
1.9)\end{array}$ & $57(9.3)$ & $54(7.6)$ & $\begin{array}{l}1.4(0.9 \\
2.1)\end{array}$ & $\begin{array}{l}0.7(0.5 \\
1.2)\end{array}$ \\
\hline \multicolumn{9}{|c|}{ Proportion of obesity in PCOS } \\
\hline $0-30 \%$ & $209(26.5)$ & $175(33.0)$ & 1 & 1 & $161(26.4)$ & $223(31.5)$ & 1 & 1 \\
\hline $31-50 \%$ & $321(40.7)$ & $220(41.5)$ & $\begin{array}{l}1.2(0.9 \\
1.6)\end{array}$ & $\begin{array}{l}1.0(0.8, \\
1.4)\end{array}$ & $248(40.8)$ & $293(41.3)$ & $\begin{array}{l}1.2(0.9 \\
1.5)\end{array}$ & $\begin{array}{l}1.0(0.8 \\
1.4)\end{array}$ \\
\hline $51-80 \%$ & $222(28.2)$ & 109 (20.6) & $\begin{array}{l}1.7(1.3 \\
2.3)^{a}\end{array}$ & $\begin{array}{l}1.4(1.0 \\
1.9)^{\mathbf{a}}\end{array}$ & $167(27.4)$ & $164(23.1)$ & $\begin{array}{l}1.4(1.1 \\
1.9)^{a}\end{array}$ & $\begin{array}{l}1.2(0.9 \\
1.6)\end{array}$ \\
\hline$>80 \%$ & $36(4.6)$ & $26(4.9)$ & $\begin{array}{l}1.2(0.7 \\
2.0)\end{array}$ & $\begin{array}{l}1.1(0.6, \\
1.9)\end{array}$ & $33(5.4)$ & $29(4.1)$ & $\begin{array}{l}1.6(0.9, \\
2.7)\end{array}$ & $\begin{array}{l}1.6(0.9, \\
2.7)\end{array}$ \\
\hline
\end{tabular}

Abbreviations: OGTT Oral glucose tolerance test, Repro-Endo Reproductive endocrinologist, OB-Gyn Obstetrician-gynecologists, PCOS Polycystic ovary syndrome a $P<0.05$

lifestyle modification (>95\%), followed by metformin (> $80 \%$ ) in both groups (Fig. 2). Participants in the ReproEndo group were more likely to prescribe metformin for patients with obesity and fertility concerns $(97.3 \%$ vs. 79.7\%, $P<0.001$ ) (Fig. 2b) than those in the OB-Gyn group.

When physicians were asked how they would advise patients with obesity and PCOS to lose weight, the most common strategy in both groups was "referral to a clinical dietitian" (87.7\% vs. $82.9 \%$ ). In addition, more Repro-Endo participants prescribed metformin than those in the OBGyn group for patients with obesity desiring weight loss $(88.8 \%$ vs. $74.3 \%, P<0.001)$. The third suggestion for weight loss in both groups was Traditional Chinese medicine (TCM), including 50.8 and $53.4 \%$ of participants in the Repro-Endo and OB-Gyn groups, respectively $(P=$
0.509) (Fig. 3a). Overall, the most common dosage of metformin was $1000 \mathrm{mg} /$ day (37.3\%), followed by $1500 \mathrm{mg} /$ day (29.3\%). More Repro-Endo participants recommended metformin at a dosage of $1500 \mathrm{mg} /$ day than OB-Gyn participants $(47.6 \%$ vs. $26.3 \%, P<0.001)$, whereas more OBGyn participants reported being unclear about the appropriate dosage of metformin for patients with obesity and PCOS than Repro-Endo participants $(8.9 \%$ vs. $1.6 \%, P=$ 0.001 ) (Fig. 3b). Moreover, only $7.7 \%$ of the participants stated they would prescribe glucagon-like peptide-1 (GLP1) receptor agonist for patients with obesity and PCOS for weight loss (Fig. 3a).

\section{Discussion}

Obesity is a common feature of PCOS, and it aggravates reproductive and metabolic abnormalities in these 


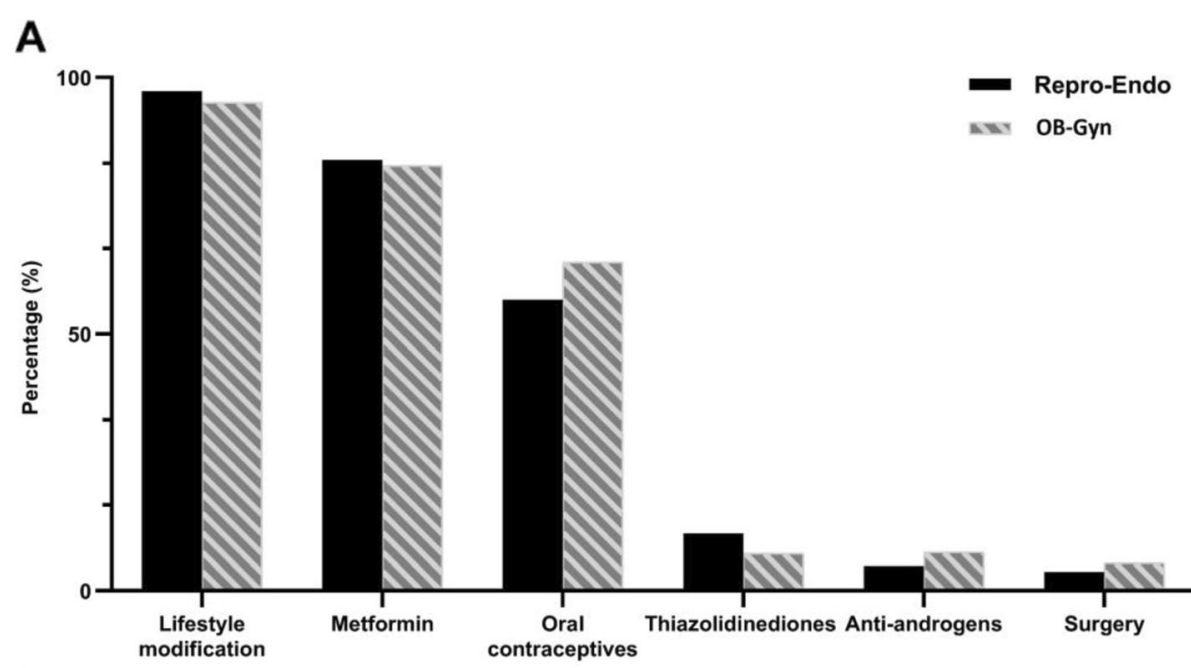

B

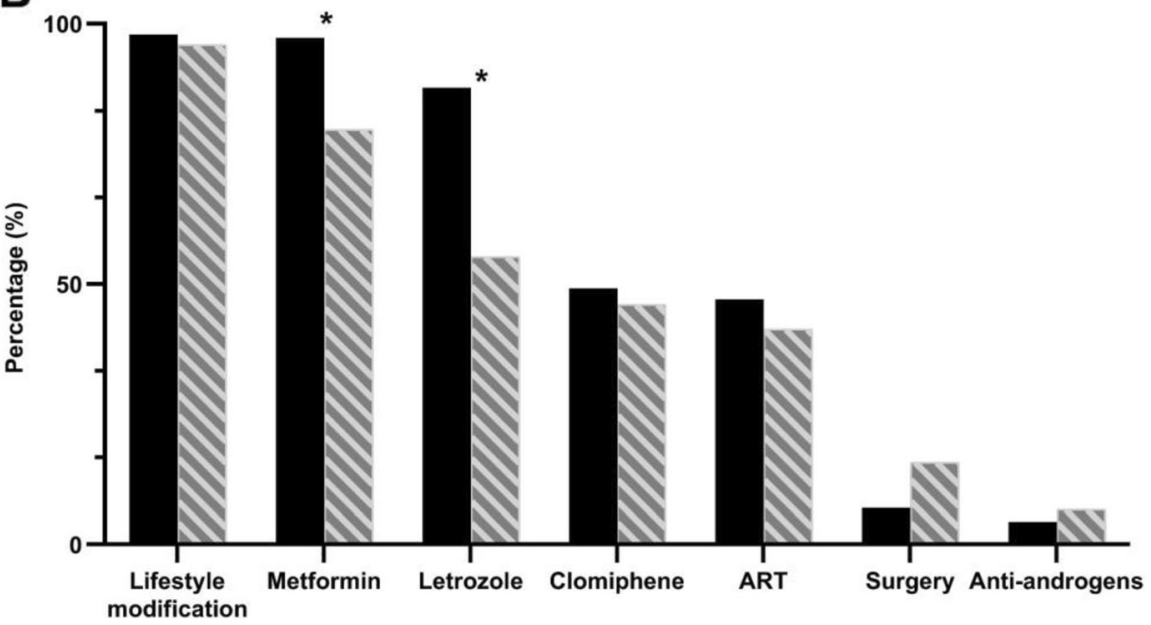

Fig. 2 Treatment recommendations for women with obesity and PCOS. a Patients with no fertility demand. b Patients with fertility demand. Repro-Endo, reproductive endocrinologist; OB-Gyn, obstetrician-gynecologists; ART, assisted reproduction technology. ${ }^{*} p<0.05$

patients. Among endocrinologists in Europe and OBgynecologists and endocrinologists in America (ACOG and American Society for Reproductive Medicine (ASRM) members), obesity was the most important long-term concern of patients with PCOS [13, 14]. To the best of our knowledge, our study is the largest survey conducted to determine the practice and pattern of Chinese physicians in the assessment and management of obesity in PCOS patients. We observed that a significant percentage of physicians did not consider abdominal obesity and psychological problems in patients with PCOS. They did not routinely conduct OGTT and lipid profile in patients with obesity and PCOS, as recommended by current guidelines [9-11]. Most participants were aware of the benefits of lifestyle modification on obesity-related symptoms and recommended patients with obesity to consult a clinical dietitian. Moreover, metformin was widely used to improve insulin sensitivity and weight loss. Our results indicate significant differences between Repro-Endo and OB-Gyn specialists in their evaluation and treatment of patients with obesity and PCOS. Similarly, this highlights the need to educate physicians caring for women with obesity and PCOS.

Most participants in this study reported BMI as a diagnostic criterion for obesity. As height and weight are easily accessible anthropometric indicators, BMI is the most widely used measure of obesity worldwide. Nonetheless, studies have shown that metabolic dysfunction in patients with obesity may not be related to weight but to abnormal fat distribution, and abdominal obesity is an important risk factor for metabolic syndrome, diabetes, and cardiovascular diseases $[16,17]$. The waistline is recognized as the most simple and practical indicator of abdominal adiposity; however, only a few participants in this survey preferred measuring the waistline of patients with obesity and PCOS. 

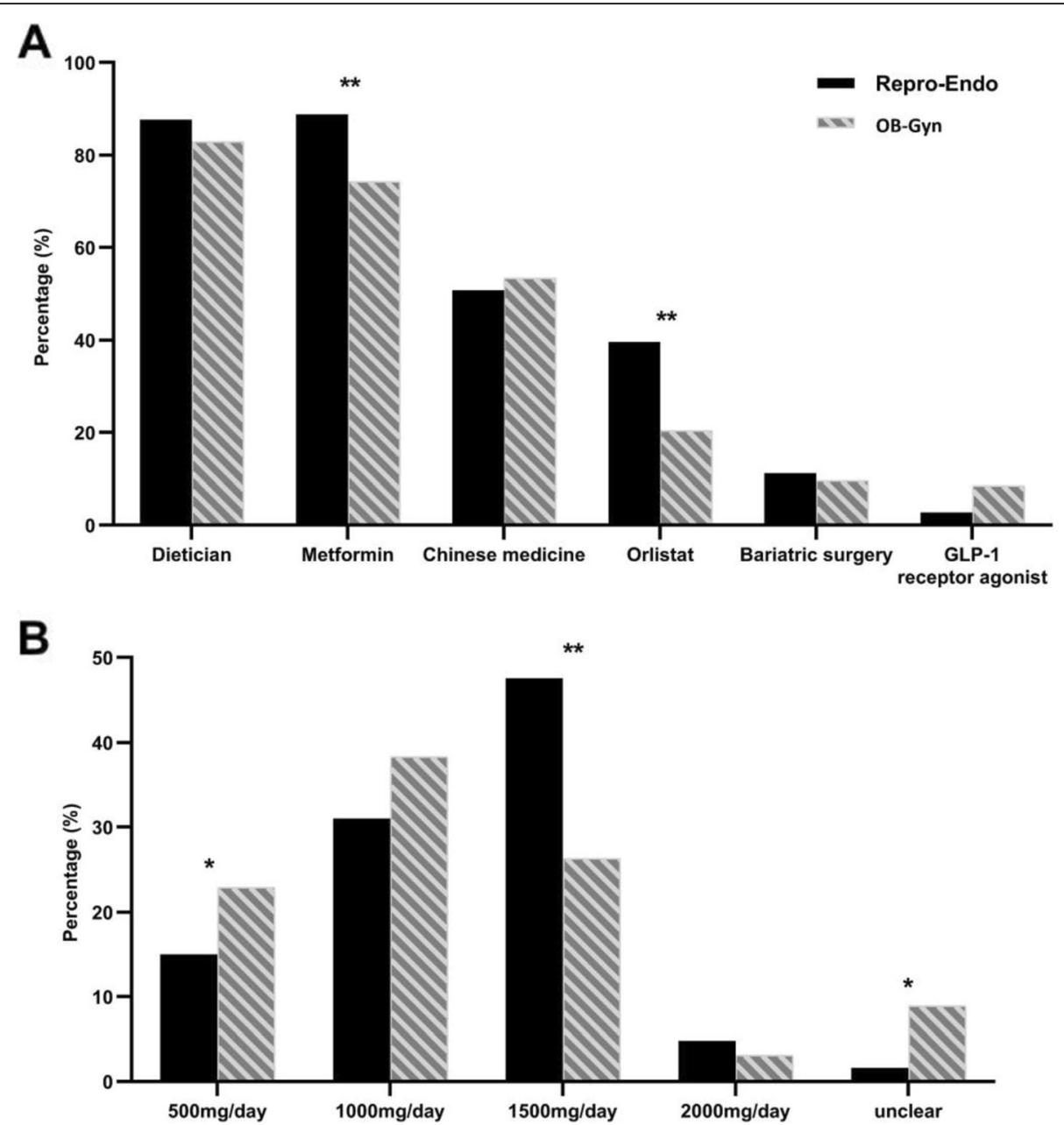

Fig. 3 Suggestions for weight loss. a Recommended way to lose weight for women with obesity and PCOS. b Most common dosage of metformin prescribed in women with obesity and PCOS. Repro-Endo, reproductive endocrinologist; OB-Gyn, obstetrician-gynecologists; GLP-1 receptor agonist, glucagon-like peptide-1 receptor agonist. ${ }^{*} p<0.05,{ }^{* *} p<0.001$

A recent meta-analysis reported that women with PCOS were more likely to develop moderate and severe depression and anxiety symptoms. Although the etiology of this association remains unclear, these symptoms weakly correlated with age, BMI, elevated testosterone levels, and IR [8]. Moreover, obesity is associated with a decline in the quality of life of women with PCOS [18]. Our study showed that more Repro-Endo participants inquired about psychological problems of women with obesity and PCOS than OB-Gyn participants; however, these providers accounted for a small proportion of all participants. However, we did not survey physicians on their practices regarding psychological counseling.

Current guidelines recommend that two-hour OGTT and fasting lipid and lipoprotein levels should be measured in patients with obesity and PCOS at the time of diagnosis to evaluate metabolic abnormalities [9-11]. Obesity substantially contributes to metabolic abnormalities in women with PCOS, as it leads to dyslipidemia,
IR, and IGT [19]. Abdominal obesity, dyslipidemia, and IGT are central components of metabolic syndrome and are notable risk factors of diabetes and cardiovascular disease [20, 21]. An online survey of ACOG members showed that metabolic screening in patients with PCOS was largely underutilized among OB-Gyn [22]. In this study, only $42.5 \%$ of participants reported ordering both a lipid profile and OGTT for patients with obesity and PCOS. Moreover, there were significant differences between the Repro-Endo and OB-Gyn participants in implementing guideline-recommended metabolic screening tests. Consequently, women with PCOS may be incompletely evaluated when seen by different specialists.

Nearly all participants reported lifestyle modification as the first-line treatment for patients with PCOS with or without fertility requirements, and most of them advised patients with obesity and PCOS to visit a clinical dietitian. This indicates a tendency toward a 
multidisciplinary strategy for obesity management. Metformin, an insulin sensitizer, is commonly used in combination with lifestyle modification to treat PCOS. Improvement in insulin sensitivity due to metformin administration is associated with its ability to decrease androgen levels, increase ovulation rate, and improve glucose tolerance [23]. Physicians in this survey generally accepted the weight-loss effect of metformin. Metformin can be safely administered at a dosage ranging from 500 to $2550 \mathrm{mg} /$ day; however, its gastrointestinal side effects are dose-related. Therefore, the dosage of metformin is increased gradually. Patients with T2DM benefit most when taking metformin at the upper recommended daily dosage [24], and patients with IR and IGT taking metformin at $1500 \mathrm{mg} /$ day benefitted from a reduction in fasting blood glucose and OGTT-2 h blood glucose levels and homeostatic model assessment of insulin resistance (HOMA-IR) index than those taking metformin at $500 \mathrm{mg} /$ day [25]; however, a few studies have compared the efficacy of different doses of metformin on PCOS. A multicenter study reported that metformin administered at $1000 \mathrm{mg} /$ day or $1500-1700 \mathrm{mg} /$ day showed similar therapeutic effects on the clinical and biochemical parameters of PCOS patients [26]. Similarly, there was evidence that higher doses of metformin resulted in better weight loss in patients with obesity and PCOS [27]. Our results indicate a disparity in knowledge regarding the use of metformin between groups, which may lead to inadequate treatment for patients with obesity and PCOS.

More than half of the participants in this survey recommended TCM therapy for weight loss. TCM therapy, as an approach of complementary and alternative medical (CAM) treatment, has been used in women with PCOS to improve ovulatory dysfunction, IR, hyperandrogenism, and hyperlipemia either in combination with modern medicine or alone [28]. Moreover, the application of acupuncture or electroacupuncture for overweight/obese women with PCOS has been a common practice in Chinese medicine and has proven helpful for patients with weight loss [29]. TCM is a promising therapy for overweight/obese patients with PCOS, and highquality trials are still needed to assess the efficacy of TCM for weight loss.

The GLP-1 receptor agonist is a new type of antidiabetic drug that has been used in the treatment of PCOS in recent years. GLP-1 receptor agonists have been shown to be effective in improving IR and IGT and are equally associated with weight loss due to delayed gastric emptying and increased satiety via a central action [30, 31]. Studies have shown that the GLP-1 receptor agonists are superior to metformin in reducing weight and improving IR [32]. Participants in this survey appeared to be less aware of GLP-1 receptor agonists.
Nevertheless, more clinical trials and cost-benefit analyses are needed to definitively guide physicians in the use of the GLP-1 receptor agonist as a treatment option for PCOS.

\section{Conclusion}

In conclusion, this survey appears to be the largest study to evaluate the patterns of practice regarding the management of obesity in PCOS patients across China. The results of this survey not only generate a clear picture of the present status of the management of obesity and PCOS, but also helps in identifying some underlying problems in practice. Nonetheless, there were some limitations to this study. First, the age and specialty of the participants were unevenly distributed. Second, only a small number of reproductive endocrinologists were noted. Finally, we did not survey physicians with respect to their practices regarding follow-up visits of patients with obesity and PCOS; additionally, we did not survey the age of patients with obesity and PCOS. Therefore, further investigations are needed to confirm and update our conclusions.

Our results identified knowledge gaps in metabolic screening for patients with obesity and PCOS as well as disparities in their evaluation and treatment among different specialties. Similarly, it highlights the potential for improvement in obesity management education for physicians caring for women with PCOS. Further research is needed to identify gaps in the care of patients with obesity and PCOS, improve the practice of physicians in the management of obesity and PCOS, and emphasize the importance of obesity prevention from childhood or adolescence.

\section{Abbreviations}

CHS: China Hypertension Survey; BMI: Body mass index; T2DM: Type 2 diabetes mellitus; PCOS: Polycystic ovary syndrome; IR: Insulin resistance; IGT: Impaired glucose tolerance; ACOG: American College of Obstetricians and Gynecologists; OGTT: Oral glucose tolerance test; Repro-

Endo: Reproductive endocrinologists; OB-Gyn: Obstetrician-gynecologists;

GLP-1 receptor agonist: Glucagon-like peptide-1 receptor agonist;

ASRM: American Society for Reproductive Medicine

\section{Supplementary Information}

The online version contains supplementary material available at https://doi. org/10.1186/s12902-021-00848-w.

\section{Additional file 1}

\section{Acknowledgements}

The authors would like to thank all Chinese obstetricians and gynecologists who participated in the survey for sharing their valuable views. We also thank China Maternal and Child Health Association for supporting our survey. We would like to thank Editage (www.editage.cn) for English language editing.

Authors' contributions

RLM and AJS conceptualized the research topic and designed the questionnaire. WW, QMZ, YF, YZ, HD, ZYT, XQZ, YQZ, YD, and YFW provided 
valuable comments and suggestions on the questionnaire revision and played a critical role in the distribution, completion, and collection of questionnaires. RLM, WW, YZ, and AJS analyzed and interpreted the data. YD and YFW provided guidance for the statistical analysis. RLM was a major contributor in writing the manuscript. AJS, RLM, and YZ were responsible for revision. All authors read and approved the final manuscript.

\section{Funding}

This work was supported by the Independent Innovation Projects of Beijing Municipal Health Commission (grant No. 2020-2-40113).

\section{Availability of data and materials}

The datasets used and/or analysed during the current study available from the corresponding author on reasonable request.

\section{Declarations}

\section{Ethics approval and consent to participate}

The study was approved by the Ethics Committee of Peking Union Medical College Hospital, Chinese Academy of Medical Sciences (No. S-K1373). Written informed consent was obtained from all participants prior to the online survey.

\section{Consent for publication}

Not applicable.

\section{Competing interests}

The authors declare that they have no competing interests.

\section{Author details}

'Department of Obstetrics and Gynecology, Peking Union Medical College Hospital, Chinese Academy of Medical Sciences and Peking Union Medical College, Beijing 100730, China. ${ }^{2}$ Department of Obstetrics and Gynecology, Hunan Provincial Maternal and Child Health Care Hospital, Changsha 410008 Hunan, China. ${ }^{3}$ Department of Reproductive Medicine, The Second Hospital of Hebei Medical University, Shijiazhuang 050000, Hebei, China. ${ }^{4}$ Department of Gynecology, The Affiliated Hospital of Qingdao University, Qingdao 266500, Shandong, China. ${ }^{5}$ Department of Obstetrics and Gynecology, The Second Affiliated Hospital of Nanchang University, Nanchang 330006, Jiangxi, China. 'Department of Obstetrics and Gynecology, Women and Children's Hospital of Jinzhou, Jinzhou 121000, Liaoning, China. 'Department of Obstetrics and Gynecology, Xinhui Maternity and Children's Hospital, Nanning 529100, Guangxi, China. ${ }^{8}$ Department of Gynecology, Guangzhou Women and Children's Medical Center, Guangzhou 510000, Guangdong, China. ${ }^{9}$ Department of Obstetrics and Gynecology, Peking Union Medical College Hospital, No. 1 Shuaifuyuan, Dongcheng District, Beijing 100010, China.

Received: 15 December 2020 Accepted: 26 August 2021

\section{Published online: 06 September 2021}

\section{References}

1. Zhang L, Wang Z, Wang $X$, Chen Z, Shao L, Tian Y, et al. Prevalence of overweight and obesity in China: results from a cross-sectional study of 441 thousand adults, 2012-2015. Obes Res Clin Pract. 2020;14(2):119-26. https:// doi.org/10.1016/j.orcp.2020.02.005.

2. $\quad$ Yang G, Wang Y, Zeng Y, Gao GF, Liang X, Zhou M, et al. Rapid health transition in China, 1990-2010: findings from the global burden of disease study 2010. Lancet. 2013;381(9882):1987-2015. https://doi.org/10.1016/S014 0-6736(13)61097-1.

3. Escobar-Morreale HF. Polycystic ovary syndrome: definition, aetiology, diagnosis and treatment. Nat Rev Endocrinol. 2018;14(5):270-84. https://doi. org/10.1038/nrendo.2018.24.

4. lughetti L, Lucaccioni L, Predieri B. Childhood obesity and environmental pollutants: a dual relationship. Acta Biomed. 2015;86(1):5-16.

5. Zhang HY, Guo CX, Zhu FF, Qu PP, Lin WJ, Xiong J. Clinical characteristics, metabolic features, and phenotype of Chinese women with polycystic ovary syndrome: a large-scale case-control study. Arch Gynecol Obstet. 2013; 287(3):525-31. https://doi.org/10.1007/s00404-012-2568-z.
6. Barber TM, Hanson P, Weickert MO, Franks S. Obesity and polycystic ovary syndrome: implications for pathogenesis and novel management strategies. Clin Med Insights Reprod Health. 2019;13:1179558119874042.

7. Behboudi-Gandevani S, Ramezani Tehrani F, Rostami Dovom M, Farahmand M, Bahri Khomami M, Noroozzadeh M, et al. Insulin resistance in obesity and polycystic ovary syndrome: systematic review and meta-analysis of observational studies. Gynecol Endocrinol. 2016;32(5):343-53. https://doi. org/10.3109/09513590.2015.1117069.

8. Cooney LG, Lee I, Sammel MD, Dokras A. High prevalence of moderate and severe depressive and anxiety symptoms in polycystic ovary syndrome: a systematic review and meta-analysis. Hum Reprod. 2017;32(5):1075-91. https://doi.org/10.1093/humrep/dex044.

9. Endocrinology S. Expert panel CSoO, Gyneocology CMA. Chinese guideline for diagnosis and management of polycystic ovary syndrome. Zhonghua Fu Chan Ke Za Zhi. 2018;53:2-6.

10. American College of Obstetricians and Gynecologists' Committee on Practice Bulletins-Gynecology. ACOG Practice Bulletin No. 194: polycystic ovary syndrome. Obstet Gynecol. 2018;131:e157-e71.

11. Teede HJ, Misso ML, Costello MF, Dokras A, Laven J, Moran L, et al. Recommendations from the international evidence-based guideline for the assessment and management of polycystic ovary syndrome. Hum Reprod. 2018;33(9):1602-18. https://doi.org/10.1093/humrep/dey256.

12. Gibson-Helm M, Dokras A, Karro H, Piltonen T, Teede HJ. Knowledge and practices regarding polycystic ovary syndrome among physicians in Europe, North America, and internationally: an online questionnaire-based study. Semin Reprod Med. 2018;36(01):19-27. https://doi.org/10.1055/s-0038-1667155.

13. Conway G, Dewailly D, Diamanti-Kandarakis E, Escobar-Morreale HF, Franks S, Gambineri A, et al. European survey of diagnosis and management of the polycystic ovary syndrome: results of the ESE PCOS special interest Group's questionnaire. Eur J Endocrinol. 2014;171(4):489-98. https://doi.org/10.1530/ EJE-14-0252

14. Dokras A, Saini S, Gibson-Helm M, Schulkin J, Cooney L, Teede H. Gaps in knowledge among physicians regarding diagnostic criteria and management of polycystic ovary syndrome. Fertil Steril. 2017;107:1380-6.e1.

15. Cussons AJ, Stuckey BG, Walsh JP, Burke V, Norman RJ. Polycystic ovarian syndrome: marked differences between endocrinologists and gynaecologists in diagnosis and management. Clin Endocrinol (Oxf). 2005; 62:289-95.

16. Escobar-Morreale HF, San Millán JL. Abdominal adiposity and the polycystic ovary syndrome. Trends Endocrinol Metab. 2007;18(7):266-72. https://doi. org/10.1016/j.tem.2007.07.003.

17. Ritchie SA, Connell JM. The link between abdominal obesity, metabolic syndrome and cardiovascular disease. Nutr Metab Cardiovasc Dis. 2007; 17(4):319-26. https://doi.org/10.1016/j.numecd.2006.07.005.

18. Greenwood EA, Pasch LA, Cedars MI, Legro RS, Huddleston HG. Association among depression, symptom experience, and quality of life in polycystic ovary syndrome. Am J Obstet Gynecol. 2018;219:279.e1.

19. Kakoly NS, Khomami MB, Joham AE, Cooray SD, Misso ML, Norman RJ, et al. Ethnicity, obesity and the prevalence of impaired glucose tolerance and type 2 diabetes in PCOS: a systematic review and meta-regression. Hum Reprod Update. 2018;24(4):455-67. https://doi.org/10.1093/humupd/ dmy007.

20. Dong J, Ni YQ, Chu X, Liu YQ, Liu GX, Zhao J, et al. Association between the abdominal obesity anthropometric indicators and metabolic disorders in a Chinese population. Public Health. 2016;131:3-10. https://doi.org/10.1016/j. puhe.2015.08.001

21. Saklayen MG. The global epidemic of the metabolic syndrome. Curr Hypertens Rep. 2018;20(2):12. https://doi.org/10.1007/s11906-018-0812-z.

22. Dhesi AS, Murtough KL, Lim JK, Schulkin J, McGovern PG, Power ML, et al. Metabolic screening in patients with polycystic ovary syndrome is largely underutilized among obstetrician-gynecologists. Am J Obstet Gynecol. 2016;215:579.e1.

23. Dumitrescu R, Mehedintu C, Briceag I, Purcărea VL, Hudita D. Metforminclinical pharmacology in PCOs. J Med Life. 2015;8(2):187-92.

24. Garber AJ, Duncan TG, Goodman AM, Mills DJ, Rohlf JL. Efficacy of metformin in type II diabetes: results of a double-blind, placebo-controlled, dose-response trial. Am J Med. 1997;103(6):491-7. https://doi.org/10.1016/ S0002-9343(97)00254-4.

25. Zhao X, Li Y, Chen M, Chen Y, Dai Y, Wang Y, et al. Effects of different doses of metformin treatment for 6 months on aberrant crypt foci in Chinese patients with impaired glucose tolerance. Eur J Cancer Prev. 2015;24(1):2736. https://doi.org/10.1097/CEJ.0000000000000078. 
26. Fulghesu AM, Romualdi D, Di Florio C, Sanna S, Tagliaferri V, Gambineri A, et al. Is there a dose-response relationship of metformin treatment in patients with polycystic ovary syndrome? Results from a multicentric study. Hum Reprod. 2012;27(10):3057-66. https://doi.org/10.1093/humrep/des262.

27. Harborne LR, Sattar N, Norman JE, Fleming R. Metformin and weight loss in obese women with polycystic ovary syndrome: comparison of doses. J Clin Endocrinol Metab. 2005;90(8):4593-8. https://doi.org/10.1210/jc.2004-2283.

28. Shen W, Jin B, Pan Y, Han Y, You T, Zhang Z, et al. The effects of traditional Chinese medicine-associated complementary and alternative medicine on women with polycystic ovary syndrome. Evid Based Complement Alternat Med. 2021;2021:6619597.

29. Yang J, Chon TY, Bauer BA. Use of acupuncture in overweight/obese women with polycystic ovary syndrome. Med Acupunct. 2019;31(5):267-8. https://doi.org/10.1089/acu.2019.1375.

30. Htike ZZ, Zaccardi F, Papamargaritis D, Webb DR, Khunti K, Davies MJ. Efficacy and safety of glucagon-like peptide-1 receptor agonists in type 2 diabetes: a systematic review and mixed-treatment comparison analysis. Diabetes Obes Metab. 2017;19(4):524-36. https://doi.org/10.1111/dom.12849.

31. Vilsbøll T, Christensen M, Junker AE, Knop FK, Gluud LL. Effects of glucagonlike peptide-1 receptor agonists on weight loss: systematic review and meta-analyses of randomised controlled trials. BMJ (Clinical research ed). 2012;344:d7771.

32. Elkind-Hirsch $\mathrm{K}$, Marrioneaux $\mathrm{O}$, Bhushan $\mathrm{M}$, Vernor $\mathrm{D}$, Bhushan $\mathrm{R}$. Comparison of single and combined treatment with exenatide and metformin on menstrual cyclicity in overweight women with polycystic ovary syndrome. J Clin Endocrinol Metab. 2008;93(7):2670-8. https://doi. org/10.1210/jc.2008-0115

\section{Publisher's Note}

Springer Nature remains neutral with regard to jurisdictional claims in published maps and institutional affiliations.

Ready to submit your research? Choose BMC and benefit from:

- fast, convenient online submission

- thorough peer review by experienced researchers in your field

- rapid publication on acceptance

- support for research data, including large and complex data types

- gold Open Access which fosters wider collaboration and increased citations

- maximum visibility for your research: over $100 \mathrm{M}$ website views per year

At $\mathrm{BMC}$, research is always in progress.

Learn more biomedcentral.com/submissions 